\title{
Stability Criterion for the maximum clearing time of a DFIG: an equal area criterion's equivalent for induction generators
}

\author{
Janailson Rodrigues Lima \\ SuperGrid Institute \\ janailson.rodrigues@supergrid-institute.com \\ Abdelkrim Benchaib \\ SuperGrid Institute \\ abdelkrim.benchaib@supergrid-institute.com \\ IBISC - Université d'Evry Val d'Essonne \\ gilney.damm@12s.centralesupelec.com \\ Emilia Nobile \\ GE Renewable Energy \\ emilia.nobile@GE.com \\ Alexander Schwery \\ GE Renewable Energy \\ alexander.schwery@GE.com
}

\section{Keywords}

$\ll$ Doubly fed induction motor $\gg, \ll$ Fault ride-through $\gg, \ll$ Electrical Machinery $\gg$.

\begin{abstract}
Stability is a primordial concern of power systems. From the generator point of view, it means that the machine must be able to attain an equilibrium state (possibly the pre-fault state) once the system is back to normal operation. For Synchronous Generators, the Equal Area Criterion (EAC) can be used to determine the maximum fault clearing time also known as critical clearing time. Such criterion is not valid for Induction Generators. In fact, only a few works aimed for a stability criterion for Doubly-Fed Induction Generators (DFIG). Since the EAC uses the power-angle profile of the synchronous machine, the work done here proposes the use of the power-slip profile of the DFIG. Using the power-slip profile of a DFIG, an analytic method for critical fault clearing time for a DFIG is developed. The analytic values calculated using the criterion are compared with values obtained through simulations showing accurate results. Hence, the method can be used as an indicator for transient stability for DFIG.
\end{abstract}

\section{Introduction}

Stability is a primordial concern of power systems. From the generator point of view, it means that the machine must be able to attain an equilibrium state (possibly the pre-fault value) once the system is back to normal operation [1]. Stability criterion for Doubly-Fed Induction Generators is assessed only by a few works ([2], [3]), while stability criterion for synchronous machines is the well-known Equal Area Criterion (EAC) as presented in [4].

The EAC is an indicator of how long the synchronous machine can endure a short-circuit and still be able to keep synchronized to the power system after the fault is cleared. It uses the power transfer vs power angle curve of the synchronous machine with the swing equation to assess the ability of the machine to remain stable after the first swing [4].

It is not possible, however, to directly apply the EAC for the Doubly Fed Induction Generator (DFIG). Partly because the machine has two different electrical frequencies (stator and rotor), and the rotor frequency can be adjusted to compensate the angle deviation (Fig. 1). In this case, the variable that will indicate stability will be the rotational speed. Using the torque-slip profile of the DFIG steady state circuit with its swing equation, the Authors in [2] have developed a stability criterion for DFIG. However, 


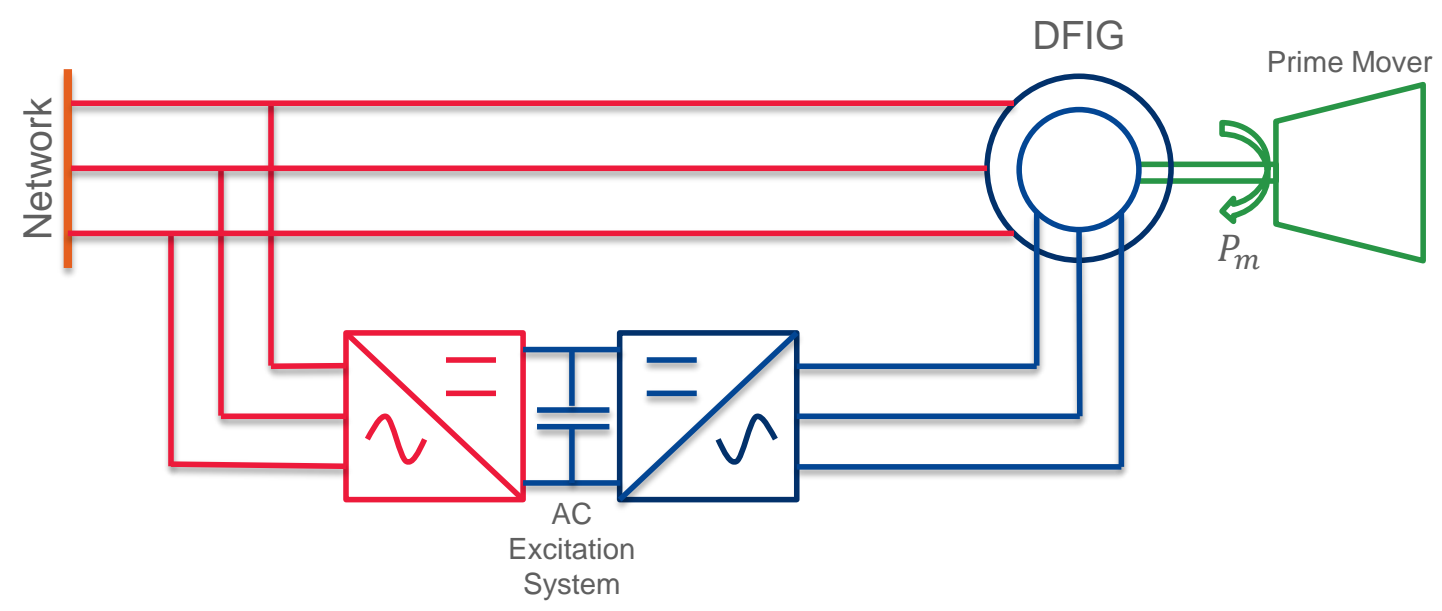

Fig. 1: Simplified scheme of the DFIG

since it is the speed deviation that will determine the stability of the machine, assume that the torque will remain constant while the speed increases is a rather strong assumption.

In the present paper, the methodology applied in [2] is expanded to the power-slip profile of the DFIG. Using data from [2] and considering the converter as ideal voltage sources, simulations were performed and compared with the results obtained through the analytic method. The results show good accuracy with the simulation, hence, the method developed here can be used as an estimation of critical clearing time in DFIG.

\section{Main result - DFIG's Stability Criterion}

The Equal Area Criterion (EAC) can be used to determine the critical clearing time for grid connected synchronous generators. However, the use of EAC for a DFIG is not possible because there is no connection between the mechanical angle of the machine and the electrical angle between the machine and the grid. To address the stability problem it is necessary to use another characteristic of the DFIG, for this purpose, the slip is used.

\section{Model and Steady State operation of DFIG}

Taking the standard DFIG model present in the literature:

$$
\begin{aligned}
& v_{s}=\frac{d \Phi_{s}}{d t}+R_{s} \cdot i_{s}+j \omega_{s} \cdot \Phi_{s} \\
& v_{r}=\frac{d \Phi_{r}}{d t}+R_{r} \cdot i_{r}+j s \omega_{s} \cdot \Phi_{r}
\end{aligned}
$$

Where the variables $\Phi, v, i$ and $s$ are, respectively, the flux, voltage, current and slip. $R$ and $\omega_{s}$ are the constants that represent the resistance and the grid frequency in rad/s. Finally, the indexes $r$ and $s$ are referent to rotor and stator, respectively. Notice, that in this model it is considered that voltage, current and flux are complex variable with the real part in the direct axis and the imaginary part in the quadrature axis.

To develop the criterion, first the steady state operation of the machine is needed. Using the dynamic model in equation (1) with zero derivatives, one has the following equations for the steady state operation of the DFIG:

$$
\begin{aligned}
& v_{s}=R_{s} \cdot i_{s}+j \omega_{s} \cdot \Phi_{s} \\
& v_{r}=R_{r} \cdot i_{r}+j s \omega_{s} \cdot \Phi_{r}
\end{aligned}
$$


Applying the definition of the flux in the stator and the rotor of the machine and considering that $X_{s}=$ $X_{s s}+X_{m}$ and $X_{r}=X_{s r}+X_{m}$, in pu, one can get to:

$$
\begin{aligned}
& j \omega_{s} X_{m}\left(i_{r}+i_{s}\right)=v_{s}-R_{s} \cdot i_{s}-j \omega_{s} X_{s S} \cdot i_{s} \\
& j \omega_{s} X_{m}\left(i_{r}+i_{s}\right)=\frac{v_{r}}{s}-\frac{R_{r}}{s} i_{r}-j \omega_{s} X_{s r} \cdot i_{r}
\end{aligned}
$$

This will lead us to the steady state circuit in Fig. 2.

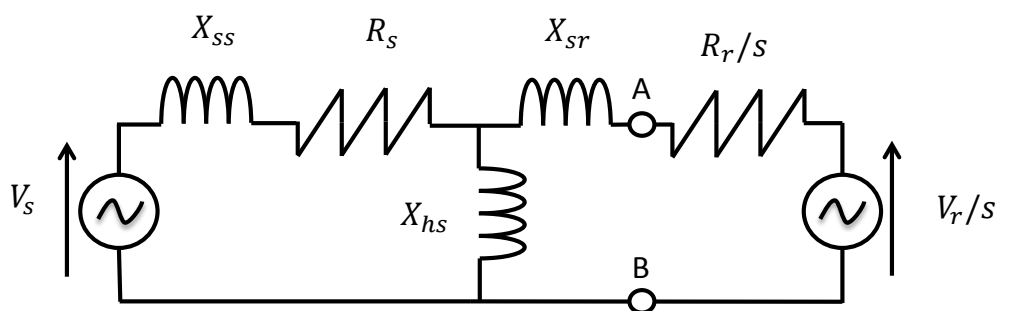

Fig. 2: Steady State circuit of the DFIG

Using the Thvenin's Theorem in points 'A' and 'B', we can develop the new steady state circuit in Fig. 3. With:

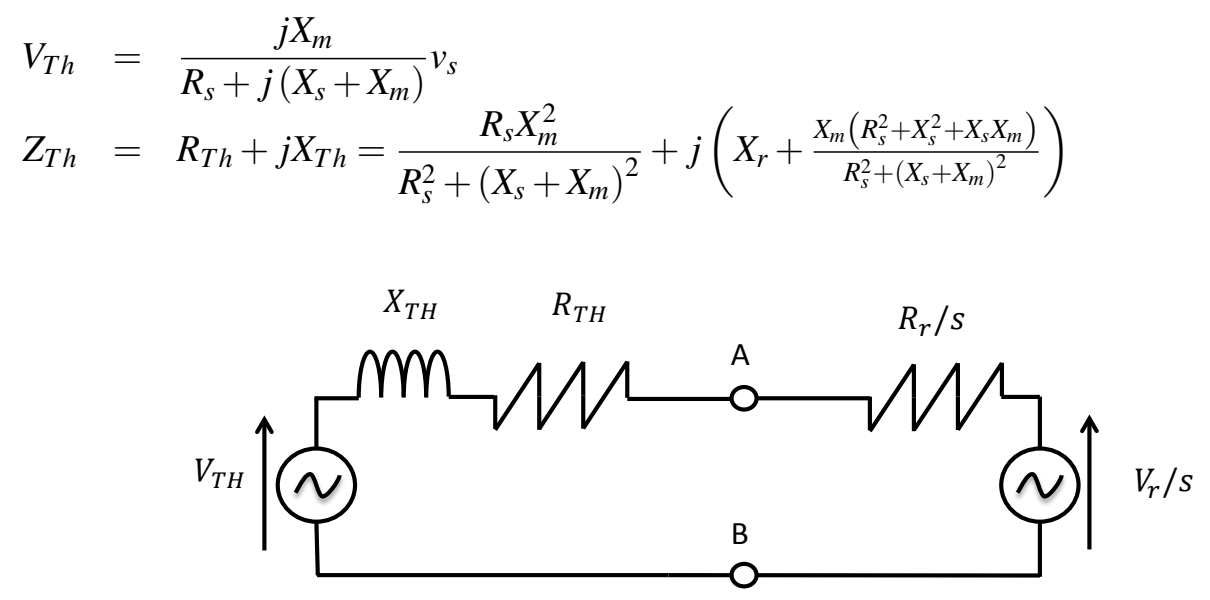

Fig. 3: Circuit of the DFIG after applying Thevenin's Theorem

So the rotor current is given by:

$$
i_{T h}=\frac{s\left(V_{T h}-v_{r}\right)}{s\left(R_{T h}+j X_{T h}\right)+R_{r}}
$$

With this circuit it is easier to calculate the electrical torque generated by the machine:

$$
T_{e}=\Phi_{r q} i_{r d}-\Phi_{r d} i_{r q}=i m\left(\Phi_{r} i_{r}^{*}\right)
$$

Applying (2):

$$
T_{e}=\frac{1}{s \omega_{s}}\left(R_{r}\left|i_{r}\right|^{2}+\operatorname{Re}\left(v_{r} \cdot i_{r}^{*}\right)\right)
$$


Considering that $P_{e}=\omega_{r} T_{e}=(1-s) \omega_{s} T_{e}$

$$
P_{m}=P_{e}=(1-s) \omega_{s} T_{e}=\frac{1-s}{s}\left(R_{r}\left|i_{r}\right|^{2}+\operatorname{Re}\left(v_{r} \cdot i_{r}^{*}\right)\right)
$$

With the power equation given by (8), it is possible to plot the power-slip characteristics of the generator Fig. 4. Moreover, it is possible to see the influence of the voltages $V_{r d}$ and $V_{r q}$ in the Power-slip profile of the DFIG (Fig. 5 and Fig. 6).

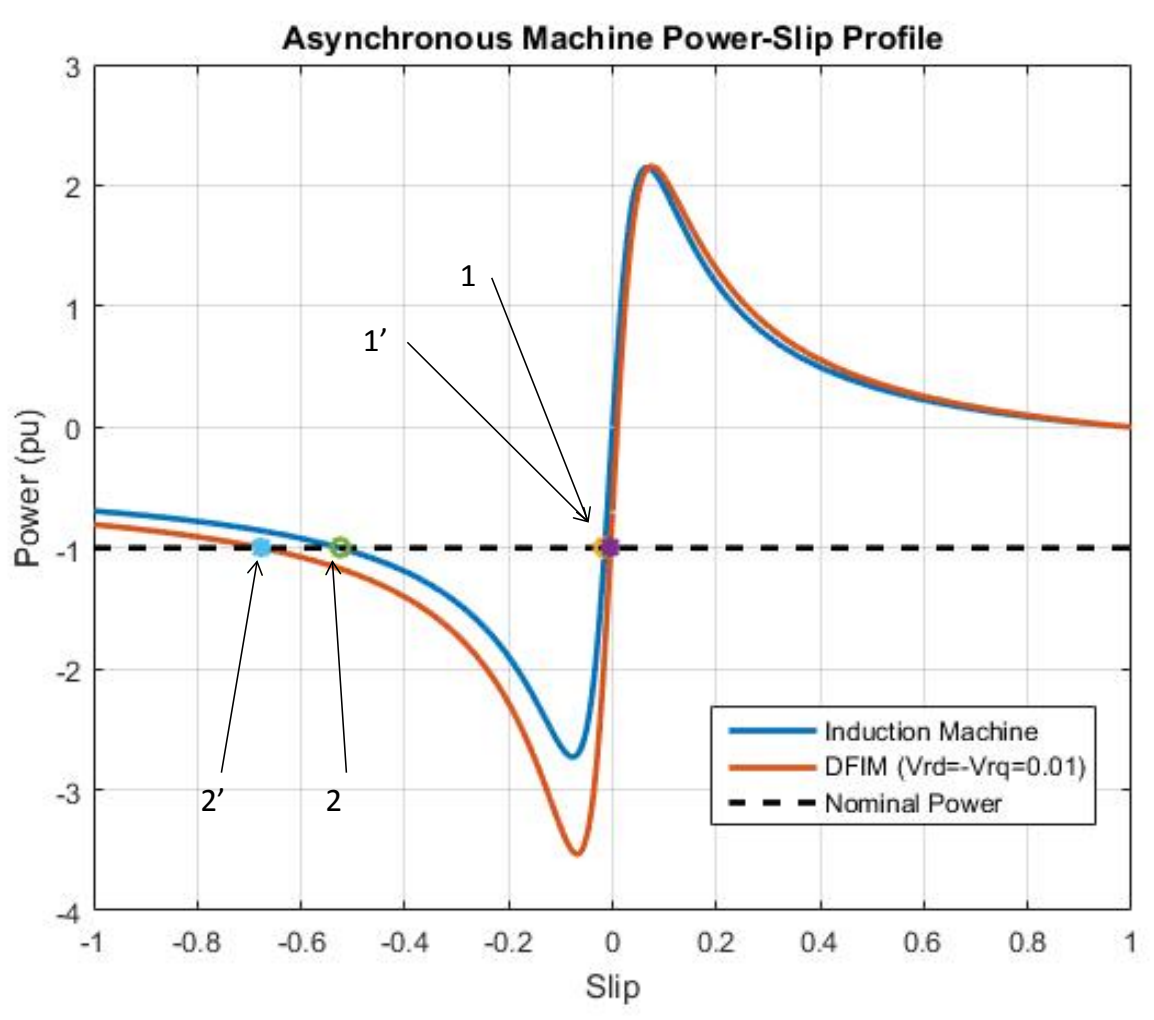

Fig. 4: Power-slip characteristic of the Induction Generator (blue) and DFIG (red)

\section{Stability criterion for induction generators}

As one can see, whether the generator has an AC excitation (DFIG) or not (Induction Generator) there are two operation points where $P_{m}=P_{e}$, however, not both of them are stable (see Fig. 4). Indeed, the operation point closest to $s=0$ is stable, while the second is not. For small perturbations at operation point 1 or 1', the power of the machine will increase or decrease in the direction of the speed, allowing the machine to come back to the initial point. For a small increase in speed at point 2 or 2', the power of the machine decreases, increasing the speed even further and so on until disconnection.

Following a short-circuit, the machine will accelerate because there is almost no voltage at its terminal. Consequently, the machine cannot send active power to the network, hence the mechanical power is converted into kinetic energy. Let's consider that before the fault, the machine was in a stable operating point 1 (asterisk point in Fig. 4). If the fault is cleared before the machine reaches point 2 (or 2'), the generator will still be able to transfer enough energy to the network such that it will reduce its own speed. If the fault takes too long to be cleared, the rotor's speed will be beyond point 2 (circle point in Fig. 4) and it will enter in the unstable region described in the previous paragraph. The critical time calculation described next is an indicator of the critical clearing time for which the machine will come back to point 1 even after a short-circuit. 


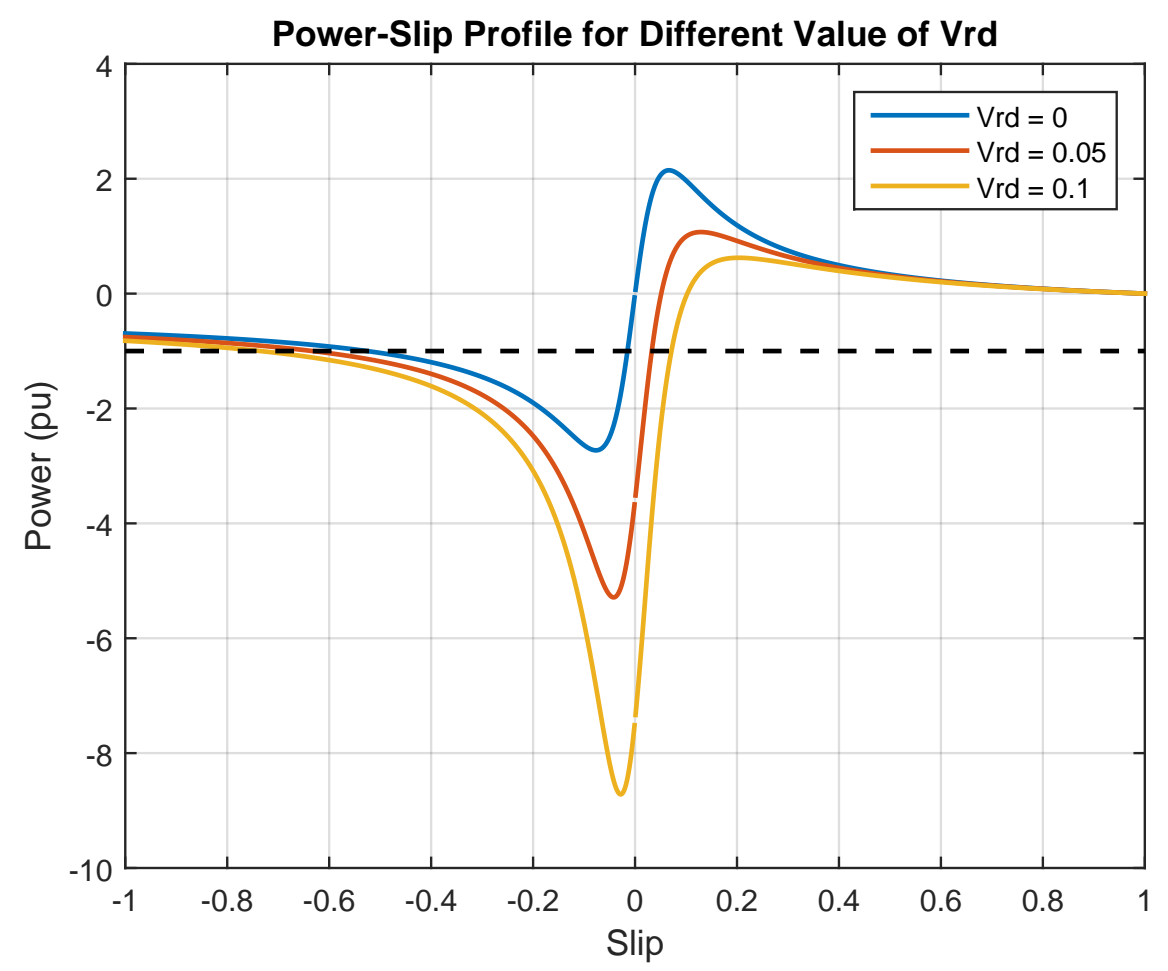

Fig. 5: Power-slip characteristic of the DFIG for different values of $V_{r d}$ and $V_{r q}=0$

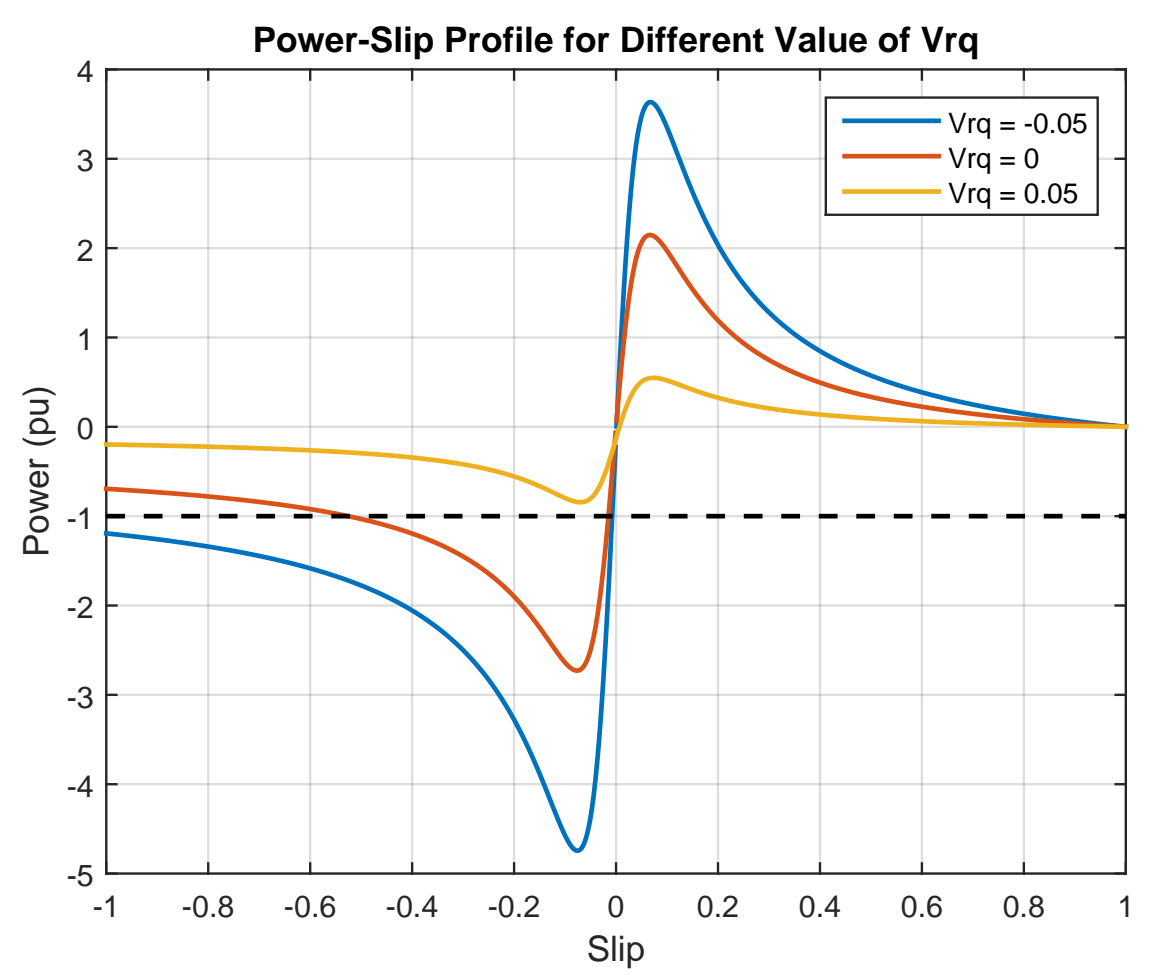

Fig. 6: Power-slip characteristic of the DFIG for different values of $V_{r d}$ and $V_{r q}=0$ 


\section{Critical Clearing Time Calculation}

From (8) a quadratic equation on the slip is developed:

$$
a_{p} s^{2}+b_{p} s+c_{p}=0
$$

Where:

$$
\begin{aligned}
& a_{p}=P_{m}\left(R_{T h}^{2}+X_{T h}^{2}\right)+R_{r}\left|V_{T h}\right|^{2}+K_{1} R_{T h}+X_{T h} K_{2} \\
& b_{p}=2 P_{m} R_{T h} R_{r}-R_{T h} K_{1}-X_{T h} K_{2}-R_{r}\left|V_{T h}\right|^{2}-R_{r} K_{1}-R_{T h}\left|v_{r}\right|^{2} \\
& c_{p}=P_{m} R_{r}^{2}+R_{T h}\left|v_{r}\right|^{2}+R_{r} K_{1} \\
& K_{1}=v_{r d} V_{T h d}+v_{r q} V_{T h q} \\
& K_{2}=v_{r d} V_{T h q}-v_{r q} V_{T h d}
\end{aligned}
$$

From (8) it is possible to obtain two operation points ( $s_{0}$ and $s_{\text {crit }}$ or $\omega_{0}$ and $\omega_{\text {crit }}$ ) by solving the equation for $s$. then, from the swing equation of the DFIG:

$$
H \frac{d \omega_{r}^{2}}{d t}=P_{m}-P_{e}
$$

Considering the worst case scenario, when the machine cannot transfer power to the network $\left(P_{e}=0\right)$. Integrating (11) from 0 to $t_{\text {crit }}$ considering the mechanical power constant and $P_{e}=0$ :

$$
H\left(\omega_{r, c r i t}^{2}-\omega_{r, 0}^{2}\right)=P_{m} t_{c r i t}
$$

Where $\omega_{r, c r i t}$ and $\omega_{r, 0}$ are the solutions of Equation (9). Thus, one can isolate $t_{c r i t}$ using the parameters of (9):

$$
t_{c r i t}=\frac{H \sqrt{b_{p}^{2}-4 \cdot a_{p} \cdot c_{p}}}{a_{p} P_{m}}\left(2+\frac{b_{p}}{a_{p}}\right)
$$

Equation (13) gives an estimation of the critical clearing time for the DFIG, as the equal area criterion allows the estimation of the critical time for synchronous machines. It is worth notice that, such as in [2], this result can be expanded to take into account the case where the machine is connected to a power network through a transmission line and with a load.

\section{Simulations and Results}

To illustrate the results of the theoretical analysis, simulations were carried out with the parameters of a DFIG applied to wind turbines as the one used in [2].

The maximum clearing time found with the simulation was compared to the time calculated using the proposed criterion in (12). The simulated maximum clearing time is given by the maximum short-circuit duration for which the machine stays connected when increasing the fault duration in steps of $10 \mathrm{~ms}$. The simulations were carried out using SIMSEN 2.3 and the comparison between the simulated and calculated results is shown in Fig. 7 and Fig. 8. 
Table I: Machine parameters used for analytic formula and simulations

\begin{tabular}{cc}
\hline \multicolumn{2}{c}{ Nominal Values } \\
\hline Power & $2(\mathrm{MVA})$ \\
Voltage & $2.4(\mathrm{kV})$ \\
Frequency & $60(\mathrm{~Hz})$ \\
\hline Machine's Parameters & \\
\hline Poles pair & 2 \\
$R_{s}$ & $0.01(\mathrm{pu})$ \\
$R_{r}$ & $0.014(\mathrm{pu})$ \\
$X_{s s}$ & $0.1(\mathrm{pu})$ \\
$X_{h s}$ & $3.5(\mathrm{pu})$ \\
$X_{s r}$ & $0.098(\mathrm{pu})$ \\
Inertia $(H)$ & $1.5(\mathrm{~s})$ \\
\hline
\end{tabular}

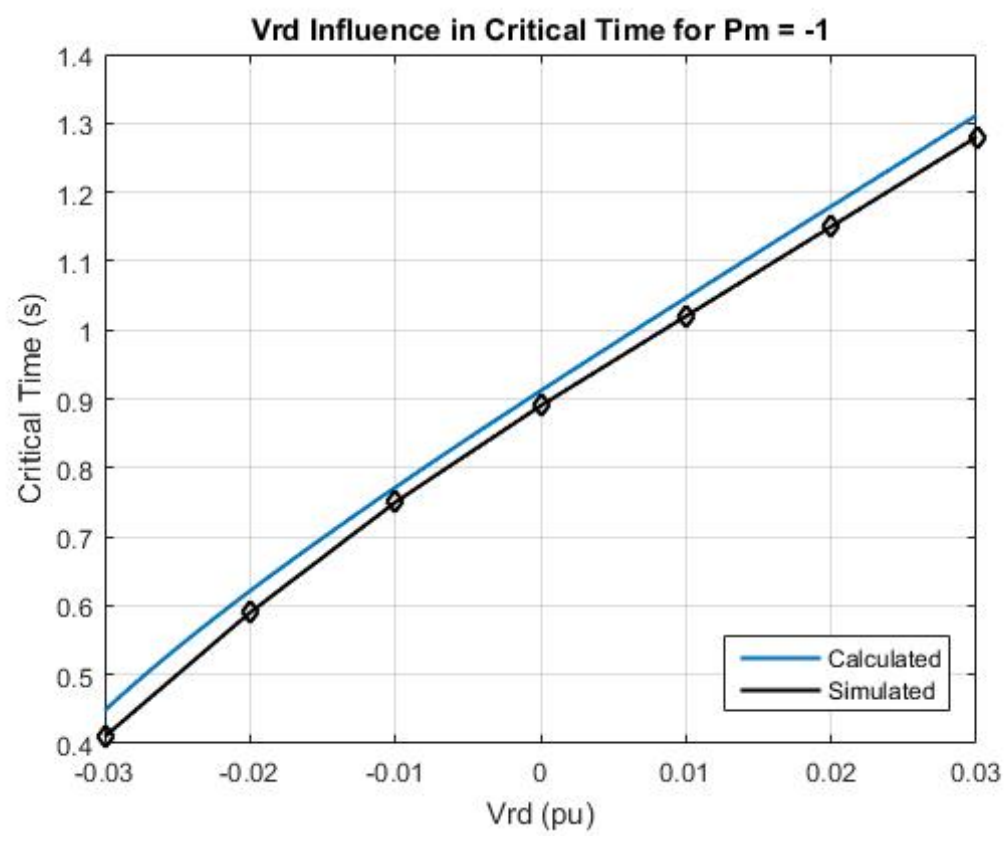

Fig. 7: Critical time versus $V_{r d}$ comparison between calculated (blue) and simulated (black) values

It is worth notice the influence of the direct and quadrature axis voltage in the value of the critical clearing time. From Fig. 7 and Fig. 8, it can be seen that a variation of quadrature axis voltage has bigger impact to the variation of the critical time than the direct axis current. This may indicate the control strategy to follow in order to protect the converter or increase the stability of the machine.

Concerning the comparison between the calculated and simulated critical time, there is a small error of about $20 \mathrm{~ms}$. The calculated time is always above the simulated one. Further investigation on the cause of this difference is needed because the calculated time is supposed to be the lower bound for the value of the critical time.

\section{Conclusion}

An analytic method to determine the critical clearing time of a DFIG is developed. Such result can be considered as the equivalent of the equal area criterion for synchronous generators, and can be used for transient stability assessment of the DFIG.

The analytic result allows a comparison between the influence of the quadrature and the direct axis voltage in the rotor's circuit of a DFIG during a fault. It has been noticed that the quadrature voltage has 


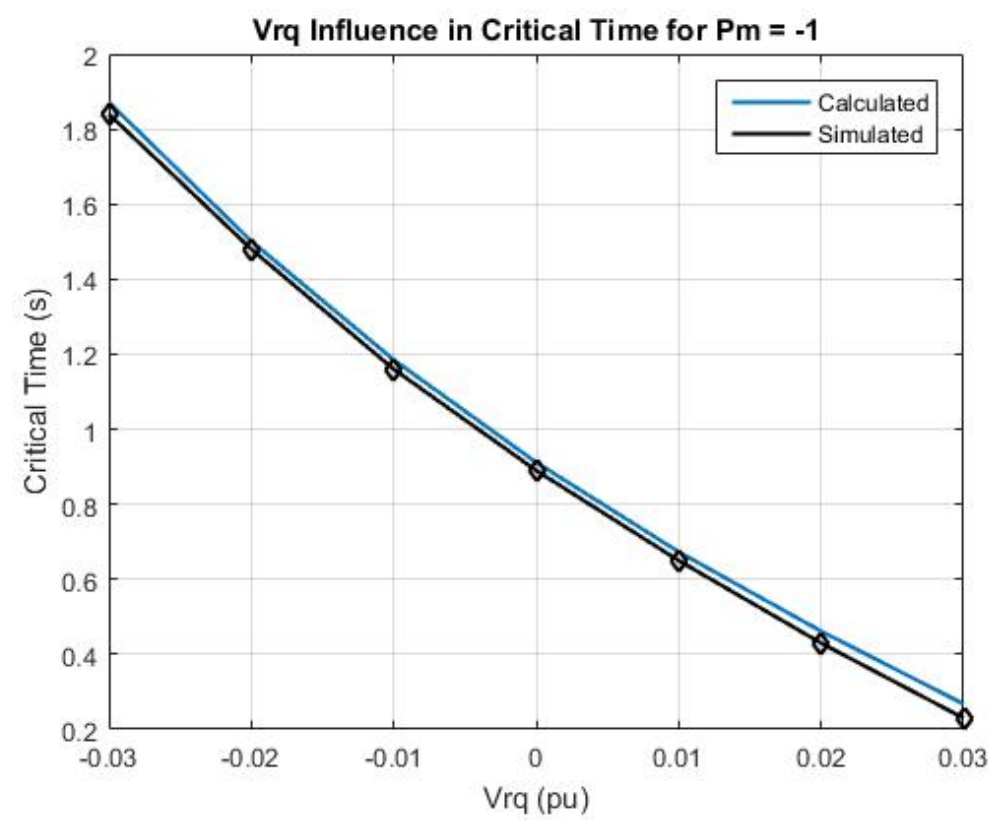

Fig. 8: Critical time versus $V_{r q}$ comparison between calculated (blue) and simulated (black) values

a bigger impact. Sensibility assessment of the formula derived here is required for further understanding this may give a better comprehension of the role played by the control of the DFIG during LVRT.

Finally, the results of the calculated critical time are compared with the maximum clearing time achieved through simulation. The calculated values are very close to the simulated ones, which means that the method developed here can be used as an indicator of the critical clearing time of a DFIG.

\section{References}

[1] IEEE/CIGRE Joint task force on stability terms and definitions, Definition and Classification of Power System Stability, 2004.

[2] Mauricio Barbosa de Camargo Salles. Modelagem e Análise de Geradores Eolicos de Velocidade Variavel Conectados em Sistemas de Energia Elétrica. PhD thesis, Escola Politécnica da Universidade de São Paulo, 2009.

[3] Mauricio B. C. Salles, Ahda P. Grilo, José Roberto Cardoso, and Ludwig Lopez Lessa. The influence of the applied rotor voltage on ride-through capability of doubly fed induction generator. In Utility Exhibition on Power and energy System: Issues \& Prospects for Asia (ICUE), Pattaya City, 2011.

[4] P. Kundur. Power System Stability and Control. New York: McGraw-Hill, 1994.

[5] André Hodder. Doubly-fed asynchronous motor-generator equipped with a 3-level VSI cascade. PhD thesis, Ecole Polytechnique Fédérale de Lausanne, 2004

[6] D. Nicolet, Y. Pannatier, B. Kawkabani, A. Schwery, F. Avellan, J.-J. Simond, and J.-L. Deniau. Transient Behavior of Variable Pump-Turbine Units. 24th Symposium on Hydraulic Machinery and Systems, October 2008.

[7] Hermes Francisco de Barros Santos La Macchia. Análise do Curto-Circuito Trifásico em geradores de Indućão Duplamente Alimentados, Master Thesis, Escola Politécnica de São Paulo, São Paulo, 2013. 